This is a postprint version of the following published document:

José A. Pereda; Ana Grande. Numerical Dispersion Relation for the 2-D LOD-FDTD Method in Lossy Media. In: IEEE Antennas and Wireless Propagation Letters, 2017, Volume 16, pp. 2122-2125 DOI: https://doi.org/10.1109/LAWP.2017.2699692

(C) 2017 IEEE. Personal use of this material is permitted. Permission from IEEE must be obtained for all other uses, in any current or future media, including reprinting/republishing this material for advertising or promotional purposes, creating new collective works, for resale or redistribution to servers or lists, or reuse of any copyrighted component of this work in other works. 


\title{
Numerical Dispersion Relation for the 2D LOD-FDTD Method in Lossy Media
}

\author{
José A. Pereda and Ana Grande
}

\begin{abstract}
A closed-form expression is derived for the numerical dispersion relation of the 2D locally one-dimensional finitedifference time-domain (LOD-FDTD) method in lossy media. In contrast to the lossless formulation, we found that transverseelectric $\left(\mathrm{TE}_{z}\right)$ and transverse-magnetic $\left(\mathrm{TM}_{z}\right)$ waves in lossy media exhibit different numerical dispersion relations. Moreover, when the material relaxation-time constant is not well resolved by the integration time-step the $\mathbf{T M}_{z}$ case shows much worse accuracy than the $\mathrm{TE}_{z}$ case. To remove this limitation, a splitfield LOD-FDTD formulation for $\mathrm{TM}_{z}$ waves is then considered which exhibits the same dispersion relation as the LOD-FDTD method for $\mathrm{TE}_{z}$ waves. The validity of the theoretical results is illustrated through numerical simulations.
\end{abstract}

Index Terms-Locally-one-dimensional finite-difference timedomain (LOD-FDTD) method, lossy media, numerical dispersion.

\section{INTRODUCTION}

Over the past 15 years there has been a growing interest in developing efficient unconditionally-stable finite-difference time-domain (FDTD) techniques such as the alternatingdirection implicit (ADI)- and the locally one-dimensional (LOD)-FDTD methods (see [1] and references within).

Both ADI- and LOD-FDTD techniques were early extended to include lossy and dispersive materials [2]-[5]. As a result of the FD approximations, these methods suffer from numerical dispersion (phase errors) and numerical dissipation (amplitude errors). Both factors can be quantified by solving the numerical dispersion relation associated to the governing FD equations. This is a requisite for a mature understanding of the operation and accuracy limits of every FDTD algorithm.

The numerical properties of the ADI-FDTD method in lossy media have been extensively studied [6]-[11]. However, to the best of our knowledge, the study of LOD-FDTD formulations in lossy media has not been addressed yet.

In this letter a closed-form expression is derived for the numerical dispersion relation of the 2D LOD-FDTD method in lossy media. Transverse-electric $\left(\mathrm{TE}_{z}\right)$ and transversemagnetic $\left(\mathrm{TM}_{z}\right)$ waves are both considered. For the sake of generality, weighted averages in time are used for discretizing the conduction terms. In contrast to what occurs in the lossless formulation, we show that $\mathrm{TE}_{z}$ and $\mathrm{TM}_{z}$ waves in lossy media exhibit different numerical dispersion relations.

Manuscript received March 17, 2017. This work was supported in part by the Spanish Government (MINECO) and the European Commission (ERDF) under Research Projects TEC2014-55463-C3-2-P and TEC2014-55463-C3-3P.

J. A. Pereda is with the Dpto. de Ingeniería de Comunicaciones (DICOM), Universidad de Cantabria, Avda. Los Castros s/n, 39005 Santander, Cantabria, Spain (email: peredaj@unican.es).

A. Grande is with the Dpto. de Electricidad y Electrónica, Universidad de Valladolid, 47011 Valladolid, Spain (email: agrande@uva.es).
For $\mathrm{TE}_{z}$ waves, we show that by properly selecting the values of the weighted-average parameters the accuracy of the formulation becomes independent of how well the material relaxation-time constant $\tau$ is resolved by the integration timestep $\Delta_{t}$. On the contrary, the same is not feasible for $\mathrm{TM}_{z}$ waves. To remove this restriction a split-field LOD-FDTD formulation is proposed. The theoretical results are validated by actual simulations.

\section{THE LOD-FDTD METHOD FOR TE $z$ WAVES}

Maxwell's curl equations for $\mathrm{TE}_{z}$ waves in isotropic and source-free media with permittivity $\epsilon$, permeability $\mu$ and conductivity $\sigma$ can be expressed as

$$
\begin{aligned}
\epsilon \frac{\partial E_{x}}{\partial t} & =\frac{\partial H_{z}}{\partial y}-\sigma E_{x} \\
\epsilon \frac{\partial E_{y}}{\partial t} & =-\frac{\partial H_{z}}{\partial x}-\sigma E_{y} \\
\mu \frac{\partial H_{z}}{\partial t} & =\frac{\partial E_{x}}{\partial y}-\frac{\partial E_{y}}{\partial x} .
\end{aligned}
$$

Following the LOD technique [12], the Crank-Nicolson scheme is first applied to (1). The resulting set of FDTD equations is then perturbed and factorized. Finally, after a further splitting into two time sub-steps, we obtain

Sub-step 1

$$
\begin{aligned}
E_{x}^{n+\frac{1}{2}}= & E_{x}^{n} \\
E_{y}^{n+\frac{1}{2}}= & E_{y}^{n}-\frac{\Delta_{t}}{2 \epsilon} \frac{\boldsymbol{\delta}_{x}}{\Delta_{x}}\left(H_{z}^{n}+H_{z}^{n+\frac{1}{2}}\right) \\
& -\frac{1}{\bar{\tau}}\left(\lambda_{1}^{(y)} E_{y}^{n}+\lambda_{2}^{(y)} E_{y}^{n+\frac{1}{2}}\right) \\
H_{z}^{n+\frac{1}{2}}= & H_{z}^{n}-\frac{\Delta_{t}}{2 \mu} \frac{\boldsymbol{\delta}_{x}}{\Delta_{x}}\left(E_{y}^{n}+E_{y}^{n+\frac{1}{2}}\right)
\end{aligned}
$$

Sub-step 2

$$
\begin{aligned}
E_{x}^{n+1}= & E_{x}^{n+\frac{1}{2}}+\frac{\Delta_{t}}{2 \epsilon} \frac{\boldsymbol{\delta}_{y}}{\Delta_{y}}\left(H_{z}^{n+\frac{1}{2}}+H_{z}^{n+1}\right) \\
& -\frac{1}{\bar{\tau}}\left(\lambda_{3}^{(x)} E_{x}^{n+\frac{1}{2}}+\lambda_{4}^{(x)} E_{x}^{n+1}\right) \\
E_{y}^{n+1}= & E_{y}^{n+\frac{1}{2}} \\
H_{z}^{n+1}= & H_{z}^{n+\frac{1}{2}}+\frac{\Delta_{t}}{2 \mu} \frac{\boldsymbol{\delta}_{y}}{\Delta_{y}}\left(E_{x}^{n+\frac{1}{2}}+E_{x}^{n+1}\right)
\end{aligned}
$$

where $\boldsymbol{\delta}_{x}$ and $\boldsymbol{\delta}_{y}$ are central-difference operators defined as in [8], $\tau=\epsilon / \sigma$ and $\bar{\tau}=\tau / \Delta_{t}$. Weighted averages have been taken for approximating the conduction terms in (2b) and (3a). We consider the weighted-average parameters subjected to the constraints $\lambda_{1}^{(y)}+\lambda_{2}^{(y)}=1$ and $\lambda_{3}^{(x)}+\lambda_{4}^{(x)}=1$. 
Note that the above LOD formulation involves 2 parameters $\lambda_{i}^{(\xi)}$ per electric field component, while 4 parameters per electric field component are needed in the ADI case [8]. The reason for this is that $E_{x}$ and $E_{y}$ are not actually split in the LOD case. Here, for comparison purposes the parameters $\lambda_{1}^{(x)}, \lambda_{2}^{(x)}, \lambda_{3}^{(y)}$ and $\lambda_{4}^{(y)}$, used in the ADI formulation, are implicitly assumed to be zero.

Following the same approach as in [8], we find that the numerical dispersion relation in the $Z$-domain for (2) and (3) is

$$
-\left(\frac{Z-1}{Z+1}\right)^{2}=\frac{\Delta_{t}^{2} K_{x}^{2}}{\mu \tilde{\epsilon}_{y}(Z)}+\frac{\Delta_{t}^{2} K_{y}^{2}}{\mu \tilde{\epsilon}_{x}(Z)}+\frac{\Delta_{t}^{2} K_{x}^{2}}{\mu \tilde{\epsilon}_{y}(Z)} \frac{\Delta_{t}^{2} K_{y}^{2}}{\mu \tilde{\epsilon}_{x}(Z)}
$$

with

$$
\begin{aligned}
& \tilde{\epsilon}_{x}(Z)=\epsilon\left(1+\frac{1}{\bar{\tau}} \frac{\lambda_{4}^{(x)} Z+\lambda_{3}^{(x)}}{Z-1}\right) \\
& \tilde{\epsilon}_{y}(Z)=\epsilon\left(1+\frac{1}{\bar{\tau}} \frac{\lambda_{2}^{(y)} Z+\lambda_{1}^{(y)}}{Z-1}\right)
\end{aligned}
$$

and

$$
K_{\xi}=\frac{1}{\Delta_{\xi}} \sin \left(\frac{\tilde{k}_{\xi} \Delta_{\xi}}{2}\right)
$$

where $\tilde{k}_{\xi}$ is the numerical wavenumber in the $\xi$-direction. Note that (4) has the same form as in the ADI case [8, eq. (12)], but with different expressions for $\tilde{\epsilon}_{x}(Z)$ and $\tilde{\epsilon}_{y}(Z)$.

By doing $Z=\exp \left(\mathrm{j} \omega \Delta_{t}\right)$ in (4), we obtain the numerical dispersion relation in the frequency domain, which reads

$$
\tan ^{2}\left(\frac{\omega \Delta_{t}}{2}\right)=\frac{\Delta_{t}^{2} K_{x}^{2}}{\mu \tilde{\epsilon}_{y}(\omega)}+\frac{\Delta_{t}^{2} K_{y}^{2}}{\mu \tilde{\epsilon}_{x}(\omega)}+\frac{\Delta_{t}^{2} K_{x}^{2}}{\mu \tilde{\epsilon}_{y}(\omega)} \frac{\Delta_{t}^{2} K_{y}^{2}}{\mu \tilde{\epsilon}_{x}(\omega)}
$$

where $\tilde{\epsilon}_{\xi}(\omega)$ is the numerical complex permittivity.

A number of FD schemes with different numerical properties can be obtained depending on the specific values of the parameters $\lambda_{i}^{(\xi)}$. A convenient choice is $\lambda_{1}^{(y)}=\lambda_{2}^{(y)}=0.5$ and $\lambda_{3}^{(x)}=\lambda_{4}^{(x)}=0.5$ which leads to

$$
\tilde{\epsilon}_{x}(\omega)=\tilde{\epsilon}_{y}(\omega)=\epsilon-j \frac{\epsilon}{2 \bar{\tau} \tan \left(\frac{\omega \Delta_{t}}{2}\right)} .
$$

As a consequence, the same dispersion relation as in the ADI case (synchronized scheme) is obtained. Moreover, with this choice, the accuracy does not depend on whether $\Delta_{t}$ resolves adequately $\tau$, as in the Crank-Nicolson method [8].

\section{THE LOD-FDTD METHOD FOR TM $z$ WAVES}

Maxwell's curl equations for $\mathrm{TM}_{z}$ waves can be expressed as

$$
\begin{aligned}
\epsilon \frac{\partial E_{z}}{\partial t} & =\frac{\partial H_{y}}{\partial x}-\frac{\partial H_{x}}{\partial y}-\sigma E_{z} \\
\mu \frac{\partial H_{x}}{\partial t} & =-\frac{\partial E_{z}}{\partial y} \\
\mu \frac{\partial H_{y}}{\partial t} & =\frac{\partial E_{z}}{\partial x} .
\end{aligned}
$$

According to the LOD-FDTD method, (9) is discretized as follows
Sub-step 1

$$
\begin{aligned}
E_{z}^{n+\frac{1}{2}}= & E_{z}^{n}+\frac{\Delta_{t}}{2 \epsilon} \frac{\boldsymbol{\delta}_{x}}{\Delta_{x}}\left(H_{y}^{n}+H_{y}^{n+\frac{1}{2}}\right) \\
& -\frac{1}{\bar{\tau}}\left(\lambda_{1} E_{z}^{n}+\lambda_{2} E_{z}^{n+\frac{1}{2}}\right) \\
H_{x}^{n+\frac{1}{2}}= & H_{x}^{n} \\
H_{y}^{n+\frac{1}{2}}= & H_{y}^{n}+\frac{\Delta_{t}}{2 \mu} \frac{\boldsymbol{\delta}_{x}}{\Delta_{x}}\left(E_{z}^{n}+E_{z}^{n+\frac{1}{2}}\right)
\end{aligned}
$$

Sub-step 2

$$
\begin{aligned}
E_{z}^{n+1}= & E_{z}^{n+\frac{1}{2}}-\frac{\Delta_{t}}{2 \epsilon} \frac{\boldsymbol{\delta}_{y}}{\Delta_{y}}\left(H_{x}^{n+\frac{1}{2}}+H_{x}^{n+1}\right) \\
& -\frac{1}{\bar{\tau}}\left(\lambda_{3} E_{z}^{n+\frac{1}{2}}+\lambda_{4} E_{z}^{n+1}\right) \\
H_{x}^{n+1}= & H_{x}^{n+\frac{1}{2}}-\frac{\Delta_{t}}{2 \mu} \frac{\boldsymbol{\delta}_{y}}{\Delta_{y}}\left(E_{z}^{n+\frac{1}{2}}+E_{z}^{n+1}\right) \\
H_{y}^{n+1}= & H_{y}^{n+\frac{1}{2}}
\end{aligned}
$$

with $\sum_{i=1}^{4} \lambda_{i}=1$. Note that now the electric field is split in time but the magnetic field is not.

Following the same approach as in the $\mathrm{TE}_{z}$ case, the dispersion relation in the $Z$-domain for (10) and (11) reads

$$
-\left(\frac{Z-1}{Z+1}\right)^{2}=\frac{\Delta_{t}^{2} K_{x}^{2}}{\mu \tilde{\epsilon}_{y}(Z)}+\frac{\Delta_{t}^{2} K_{y}^{2}}{\mu \tilde{\epsilon}_{x}(Z)}+\frac{\Delta_{t}^{2} K_{x}^{2}}{\mu \epsilon} \frac{\Delta_{t}^{2} K_{y}^{2}}{\mu \epsilon} \frac{1}{N(Z)}
$$

where

$$
N(Z)=1+\frac{\left(\lambda_{2}+\lambda_{4}\right) Z+\lambda_{1}+\lambda_{3}}{\bar{\tau}(Z-1)}+\frac{\lambda_{2} \lambda_{4} Z-\lambda_{1} \lambda_{3}}{\bar{\tau}^{2}(Z-1)}
$$

and

$$
\begin{aligned}
\tilde{\epsilon}_{x}(Z) & =\epsilon \frac{N(Z)}{1+\frac{\lambda_{2} Z-\lambda_{1}}{\bar{\tau}(Z+1)}} \\
\tilde{\epsilon}_{y}(Z) & =\epsilon \frac{N(Z)}{1+\frac{\lambda_{4} Z-\lambda_{3}}{\bar{\tau}(Z+1)}} .
\end{aligned}
$$

By doing $Z=\exp \left(\mathrm{j} \omega \Delta_{t}\right)$ in (12), we obtain the following frequency-domain dispersion relation

$$
\tan ^{2}\left(\frac{\omega \Delta_{t}}{2}\right)=\frac{\Delta_{t}^{2} K_{x}^{2}}{\mu \tilde{\epsilon}_{y}(\omega)}+\frac{\Delta_{t}^{2} K_{y}^{2}}{\mu \tilde{\epsilon}_{x}(\omega)}+\frac{\Delta_{t}^{2} K_{x}^{2}}{\mu \epsilon} \frac{\Delta_{t}^{2} K_{y}^{2}}{\mu \epsilon} \frac{1}{N(\omega)}
$$

It is worth noting that (15) is exactly the same as the dispersion relation previously obtained for the ADI-FDTD method in the case of $\mathrm{TM}_{z}$ waves [9, eq. (4)]. Therefore, all the discussion carried out in [9] applies to the present case and will not be repeated here. A common choice for the weighted-average parameters is $\lambda_{1,2,3,4}=0.25$. However, we recall that, independently of the weighted-average parameter choice, to achieve good accuracy $\tau$ must be well resolved by $\Delta_{t}$. To overcome this limitation a split-field formulation is proposed in the next section. 


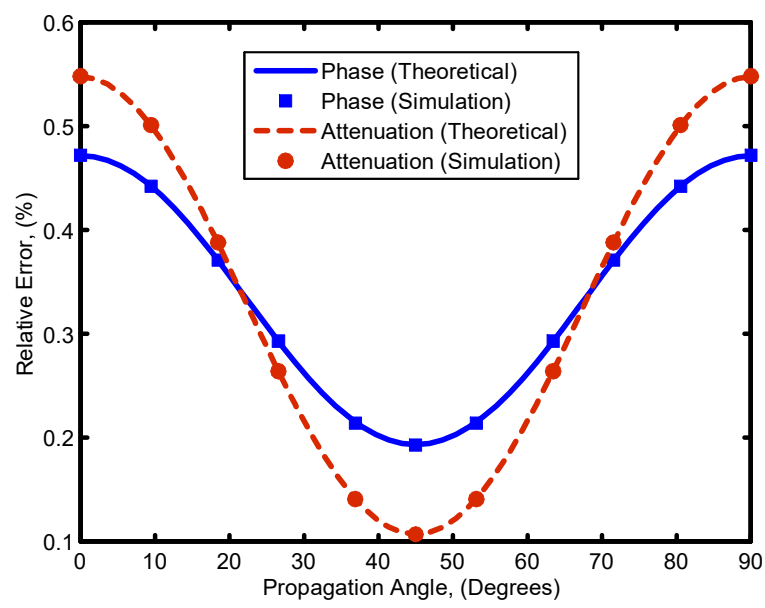

Fig. 1. Relative phase and attenuation errors against the propagation angle. Results calculated by the LOD-FDTD method for $\mathrm{TE}_{z}$ waves with $\sigma=1.8$ $\mathrm{S} / \mathrm{m}, N_{s}=40$ and $N_{t}=20$.

\section{A SPLIT-FIELD LOD-FDTD METHOD FOR TM $z$ WAVES}

Consider the following split-field version of (9) as governing equations:

$$
\begin{aligned}
\epsilon \frac{\partial E_{z x}}{\partial t} & =\frac{\partial H_{y}}{\partial x}-\sigma E_{z x} \\
\epsilon \frac{\partial E_{z y}}{\partial t} & =-\frac{\partial H_{x}}{\partial y}-\sigma E_{z y} \\
\mu \frac{\partial H_{x}}{\partial t} & =-\frac{\partial}{\partial y}\left(E_{z x}+E_{z y}\right) \\
\mu \frac{\partial H_{y}}{\partial t} & =\frac{\partial}{\partial x}\left(E_{z x}+E_{z y}\right)
\end{aligned}
$$

with $E_{z x}+E_{z y}=E_{z}$. Note that the above equations coincide with the Berenger split-field approach when in the latter the magnetic conductivities are set equal to zero [4], [13].

Applying the LOD-FDTD method to (16), the following set of difference equations is obtained:

Sub-step 1

$$
\begin{aligned}
E_{z x}^{n+\frac{1}{2}}= & E_{z x}^{n}+\frac{\Delta_{t}}{2 \epsilon} \frac{\boldsymbol{\delta}_{x}}{\Delta_{x}}\left(H_{y}^{n}+H_{y}^{n+\frac{1}{2}}\right) \\
& -\frac{1}{2 \bar{\tau}}\left(E_{z x}^{n}+E_{z x}^{n+\frac{1}{2}}\right) \\
E_{z y}^{n+\frac{1}{2}}= & E_{z y}^{n} \\
H_{x}^{n+\frac{1}{2}}= & H_{x}^{n} \\
H_{y}^{n+\frac{1}{2}}= & \frac{\Delta_{t}}{2 \mu} \frac{\boldsymbol{\delta}_{x}}{\Delta_{x}}\left(E_{z x}^{n}+E_{z x}^{n+\frac{1}{2}}+E_{z y}^{n}+E_{z y}^{n+\frac{1}{2}}\right) \\
& +H_{y}^{n}
\end{aligned}
$$

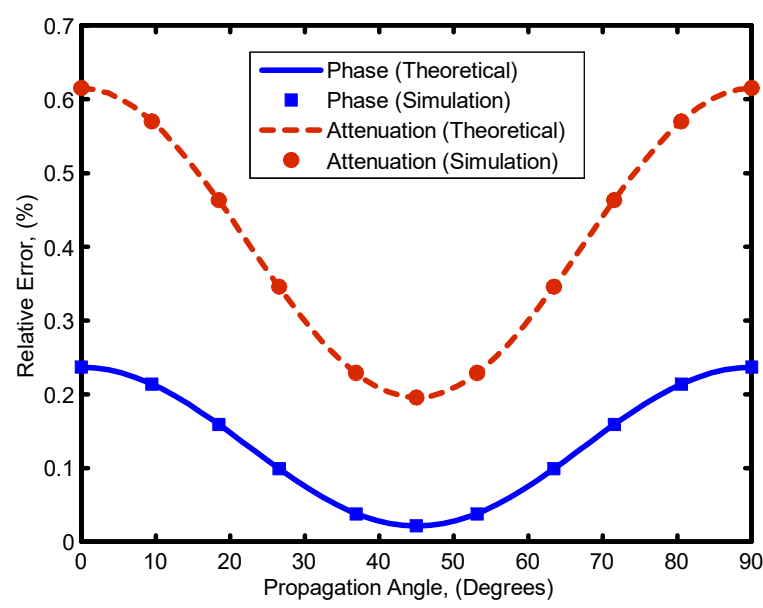

Fig. 2. Relative phase and attenuation errors against the propagation angle Results calculated by the LOD-FDTD method for $\mathrm{TE}_{z}$ waves with $\sigma=18$ $\mathrm{S} / \mathrm{m}, N_{s}=40$ and $N_{t}=20$.

\section{Sub-step 2}

$$
\begin{aligned}
E_{z x}^{n+1}= & E_{z x}^{n+\frac{1}{2}} \\
E_{z y}^{n+1}= & E_{z y}^{n+\frac{1}{2}}-\frac{\Delta_{t}}{2 \epsilon} \frac{\boldsymbol{\delta}_{y}}{\Delta_{y}}\left(H_{x}^{n+\frac{1}{2}}+H_{x}^{n+1}\right) \\
& -\frac{1}{2 \bar{\tau}}\left(E_{z y}^{n+\frac{1}{2}}+E_{z y}^{n+1}\right) \\
H_{x}^{n+1}= & -\frac{\Delta_{t}}{2 \mu} \frac{\boldsymbol{\delta}_{y}}{\Delta_{y}}\left(E_{z x}^{n+\frac{1}{2}}+E_{z x}^{n+1}+E_{z y}^{n+\frac{1}{2}}+E_{z y}^{n+1}\right) \\
& +H_{x}^{n+\frac{1}{2}} \\
H_{y}^{n+1}= & H_{y}^{n+\frac{1}{2}} .
\end{aligned}
$$

It is worth noting that in practice (17) and (18) reduce to a one step formulation. The numerical dispersion relation and the numerical permittivity for this formulation are found to be the same as the ones given in (7) and (8), respectively. Therefore, as was discussed in section II, the condition $\Delta_{t} \ll \tau$ is not required to achieve high accuracy.

\section{NUMERICAL RESULTS}

With the aim of illustrating the validity of the above theoretical results, the numerical phase and attenuation constants in a homogeneous lossy material have been calculated. The procedure followed has been the same as the one described in [14] and previously used in [8] and [9]. All the results have been calculated at the frequency $f=10 \mathrm{GHz}$. The permittivity and permeability of the lossy material are the same as in the free space. Square cells with $\Delta_{x}=\Delta_{y}=\Delta$ have been used for discretizing the computational domain. The spatial resolution was defined as $N_{s}=\lambda_{c} / \Delta$, being $\lambda_{c}$ the exact wavelength in the lossy material (at $10 \mathrm{GHz}$ ). In the same way, the temporal resolution was defined as $N_{t}=T / \Delta_{t}$, being $T=1 / f$ the period of the wave.

Figs. 1 and 2 display the relative errors for phase and attenuation against the propagation angle for $\mathrm{TE}_{z}$ waves with parameters $\lambda_{1}^{(y)}=\lambda_{2}^{(y)}=\lambda_{3}^{(x)}=\lambda_{4}^{(x)}=0.5$ and resolutions 


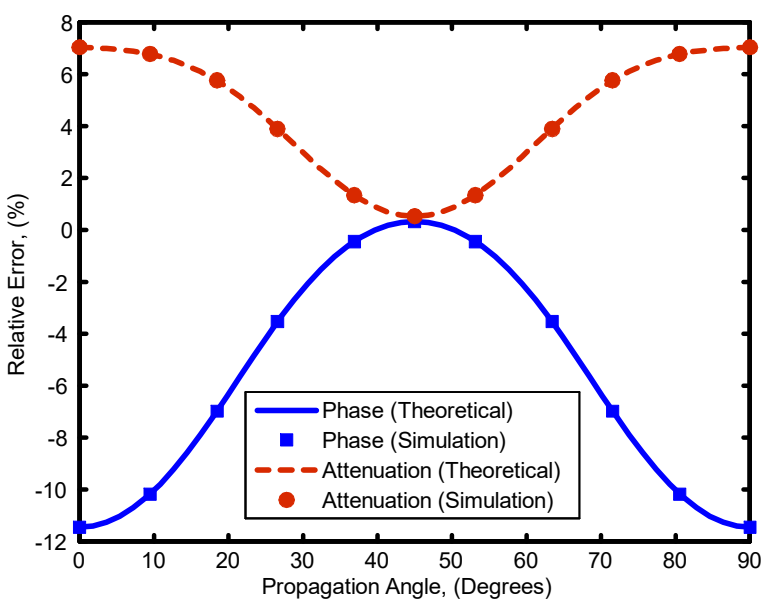

Fig. 3. Relative phase and attenuation errors against the propagation angle Results calculated by the LOD-FDTD method for $\mathrm{TM}_{z}$ waves with $\sigma=18$ $\mathrm{S} / \mathrm{m}, N_{s}=40$ and $N_{t}=20$.

$N_{s}=40$ and $N_{t}=20$. The conductivity in Fig. 1 was $\sigma=1.8$ $\mathrm{S} / \mathrm{m}$, consequently $\tau \simeq \Delta_{t}$ and $\Delta_{t} \simeq 4 \Delta_{t 0}$, where $\Delta_{t 0}$ is the maximum stable time step allowed by the conventional FDTD method. In Fig. 2 the conductivity was $\sigma=18 \mathrm{~S} / \mathrm{m}$, so in this case $\tau \simeq \Delta_{t} / 10$ and $\Delta_{t} \simeq 12 \Delta_{t 0}$. It can be seen that all the errors remain under $0.7 \%$ even though $\Delta_{t}$ is 10 times greater than $\tau$ in Fig. 2. The theoretical results obtained by directly solving (7) have been plotted by lines and those computed by actual field simulations have been denoted by markers. Excellent agreement is observed between theory and simulation. In fact, the difference between both sets of results is typically within $10^{-5} \%$.

Fig. 3 depicts the same case as in Fig. 2 but for $\mathrm{TM}_{z}$ waves with $\lambda_{1,2,3,4}=0.25$. Now very high phase and attenuation errors can be seen for angles outside the diagonal $\left(45^{\circ}\right)$.

Finally, in Fig. 4 the same example as in Fig. 2 is considered again. The difference is that in Fig. 4 the results have been obtained by using the proposed split-field LOD-FDTD method for $\mathrm{TM}_{z}$ waves. It can be seen that these results replicate those obtained in Fig. 2 for the $\mathrm{TE}_{z}$ case.

\section{CONCLUSION}

The numerical dispersion relation for the 2D LOD-FDTD method in lossy media has been derived in a closed-form. For $\mathrm{TE}_{z}$ waves the numerical dispersion relation is, in general, different from that of the ADI case. However, if central averages are used to approximate the conduction terms the dispersion relation becomes the same as in the synchronizedscheme-ADI case [8]. A salient feature of this choice is that the condition $\Delta_{t} \ll \tau$ is not required in order to achieve high accuracy. For $\mathrm{TM}_{z}$ waves, both LOD and ADI formulations exhibit an identical numerical dispersion relation. Unfortunately, for any choice of the average parameters, the condition $\Delta_{t} \ll \tau$ is necessary to achieve high accuracy. To overcome this limitation we have proposed a split-field $\mathrm{TM}_{z}$ formulation that exhibits the same numerical dispersion relation as for $\mathrm{TE}_{z}$ waves.

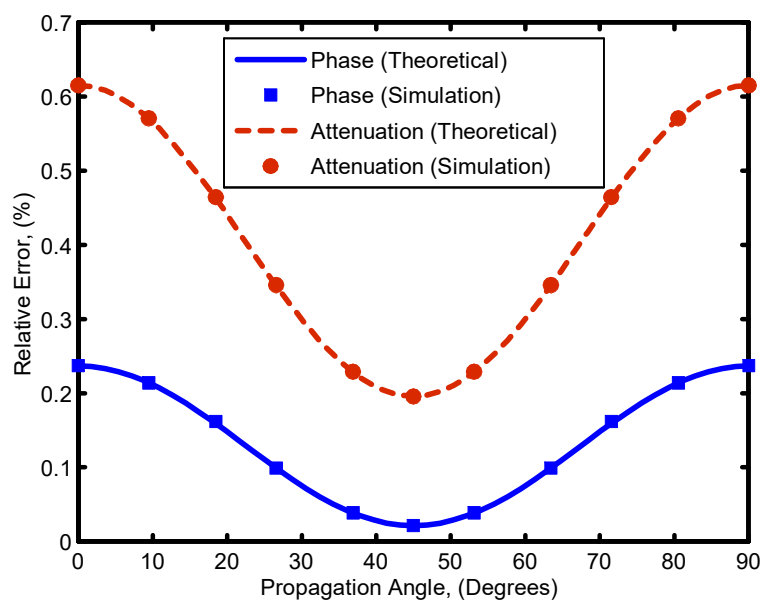

Fig. 4. Relative phase and attenuation errors against the propagation angle Results calculated by the split-field LOD-FDTD method for $\mathrm{TM}_{z}$ waves with $\sigma=18 \mathrm{~S} / \mathrm{m}, N_{s}=40$ and $N_{t}=20$.

\section{REFERENCES}

[1] J. Shibayama, M. Muraki, R. Takahashi, J. Yamauchi, and H Nakano,"Performance evaluation of several implicit FDTD methods for optical waveguide analyses," J. Lightw. Technol., vol. 24, no. 6, pp. 2465-2472, Jun. 2006.

[2] C. Yuan and Z. Chen, "On the modeling of conducting media with the unconditionally stable ADI-FDTD method," IEEE Trans. Microw. Theory Tech., vol. 51, pp. 1929-1938, Aug. 2003.

[3] S. G. García, R. G. Rubio, A. R. Bretones, and R. G. Martín, "Extension of the ADI-FDTD method to Debye media," IEEE Trans. Antennas Propag., vol. 51, pp. 3183-3186, Nov. 2003.

[4] V. E. do Nascimento, B.-H. V. Borges, and F. L. Texeira, "Splitfield PML implementation for the unconditionally stable LOD-FDTD method," IEEE Microw. Wireless Compon. Lett., vol. 16, no. 7, pp. 398 400, Jul. 2006

[5] J. Shibayama, R. Takahashi, J. Yamauchi, and H. Nakano, "Frequency dependent LOD-FDTD implementations for dispersive media," Electron. Lett., vol. 42, no. 19, pp. 1084-1085, Sep. 2006

[6] S. Wang and J.H. Duyn, "A split-field iterative ADI method for simulating transverse-magnetic waves in lossy media," IEEE Trans. Microw. Theory Tech., vol. 54, pp. 2169-2202, May 2006

[7] W. Fu and E. L. Tan, "Stability and dispersion analysis for ADI-FDTD method in lossy media," IEEE Trans. Antennas Propag., vol. 55, pp. 1095-1102, Apr. 2007

[8] J. A. Pereda, A. Grande, O. González, and A. Vegas, "Analysis of two alternative ADI-FDTD formulations for transverse-electric waves in lossy materials," IEEE Trans. Antennas Propag., vol. 57, pp. $2047-$ 2054, Jul. 2009

[9] J. A. Pereda, A. Grande, O. González, and A. Vegas, "The ADI-FDTD method for transverse-magnetic waves in conductive materials," IEEE Trans. Antennas Propag., vol. 58, pp. 2790-2793, Aug. 2010.

[10] D.Y. Heh and E.L. Tan, "Lyapunov and matrix norm stability analysis of ADI-FDTD schemes for doubly lossy media," IEEE Trans. Antennas Propag., vol. 59, pp. 979-986, Mar. 2011.

[11] T. Tan and Q. H. Liu, "Unconditionally stable ADI/Crank-Nicolson implementation and lossy split error revisited," IEEE Trans. Antennas Propag., vol. 61, pp. 5627-5636, Nov. 2013.

[12] J. Shibayama, M. Muraki, J. Yamauchi, and H. Nakano, "Efficient implicit FDTD algorithm based on locally one-dimensional scheme," Electron. Lett., vol. 41, no. 19, pp. 1046-1047, Sep. 2005.

[13] J. P. Berenger, "A perfectly matched layer for the absorption of electromagnetic waves," J. Comput. Physics, vol. 114, pp. 185-200, Oct. 1994.

[14] S. Ju, H. Kim, and H.-H. Kim, "A study of the numerical dispersion relation for the 2-D ADI-FDTD method," IEEE Microwave Wireless Comp. Lett., vol. 13, pp. 405-407, Sept. 2003. 